\title{
LIMITAÇÕES NO AVANÇO DO PROCESSO DE CARBONATAÇÃO
}

\author{
SILVA, MATHEUS DE SOUZA \\ Graduando em Engenharia Civil \\ Faculdade Ari de Sá - FAS \\ Ceará; Brasil \\ matheus.engcv@outlook.com
}

\author{
CAVALCANTE, FELIPE VASCONCELOS \\ Graduando em Engenharia Civil \\ Faculdade Ari de Sá - FAS \\ Ceará; Brasil \\ Felipevasc21@outlook.com
}

\author{
RIBEIRO, FRANCISCO ROGER CARNEIRO \\ Mestrando em Engenharia Civil \\ Universidade Vale do Rio dos Sinos - UNISINOS \\ Rio Grande do Sul; Brasil \\ roger.ribeiro_@hotmail.com
}

\author{
BRASILEIRO, FRANCISCA LILIAN CRUZ \\ Docente Mestre em Engenharia Civil \\ Faculdade Ari de Sá - FAS \\ Ceará; Brasil \\ lilian.brasileiro@outlook.com
}

\author{
BARROSO, ANNA JÉSSICA DE OLIVEIRA \\ Graduanda em Engenharia Civil \\ Faculdade Ari de Sá - FAS \\ Ceará; Brasil \\ annajessik@hotmail.com
}

\section{RESUMO}

As estruturas de concreto inseridas em meio urbano estão submetidas à degradação por efeito de agentes agressivos do ar, em especial o fenômeno de carbonatação, uma reação entre o dióxido de carbono $\left(\mathrm{CO}_{2}\right)$ e o hidróxido de cálcio $\left[\mathrm{Ca}(\mathrm{OH})_{2}\right]$ proveniente da hidratação do cimento e de outros sólidos carbonatáveis presentes nos poros do concreto. Tal reação promove a redução do $\mathrm{pH}$ do concreto, proporcionando um ambiente ácido e propício para o fenômeno de corrosão. Em vista de monitorar tal fenômeno, o meio científico desenvolveu equações de predição a fim de analisar o avanço da penetração do efeito patológico ao longo do tempo, no entanto tais formulações não apresentam valores de profundidade de carbonatação próximos aos valores reais, demonstrando imprecisão no diagnóstico de estruturas carbonatadas. Em relação a esta problemática, o objetivo desse trabalho é entender as limitações do avanço da carbonatação e inseri-lo em um sistema equacional de predição a partir da interpolação entre formulações propostas, a fim de dispor de valores mais próximos da curva de crescimento real de carbonatação de estruturas inseridas no meio urbano. Para que a análise seja possível, foram produzidos 12 corpos de prova cilíndricos $(10 \mathrm{~cm} \times 20 \mathrm{~cm})$ os quais foram submetidos a carbonatação natural durante os períodos de 14, 28, 56 e 84 dias, a fim de avaliar o comportamento do avanço da carbonatação. Os resultados analisados demonstram que os valores obtidos pela interpolação das modelagens propostas por diferentes autores apresentam aproximação superior em relação ao uso das modelagens isoladas e apresentam distanciamento máximo em relação aos valores reais inferiores a $20 \%$.

Palavras-chave: durabilidade, carbonatação, predição, eficiência.

\section{ABSTRACT}

Concrete structures inserted in an urban environment are subjected to degradation by the effects of aggressive air agents, in particular the carbonation phenomenon, a reaction between carbon dioxide $(\mathrm{CO} 2)$ and calcium hydroxide $[\mathrm{Ca}$ $(\mathrm{OH})_{2}$ f from hydration of cement and other carbonable solids present in the pores of concrete. Such a reaction promotes the reduction of the $\mathrm{pH}$ of the concrete, providing an acidic environment conducive to the phenomenon of corrosion. In order to monitor such phenomenon, the scientific community developed prediction equations in order to analyze the progress of the penetration of the pathological effect over time, however such formulations do not present carbonation depth values close to the real values, demonstrating inaccuracy in the diagnosis of carbonated structures. In relation to this problem, the objective of this work is to understand the limitations of the advance of carbonation and to insert it in an equational prediction system based on the interpolation between proposed formulations, in order to have values closer to the real growth curve of carbonation of structures inserted in the urban environment. In order for the analysis to be possible, 12 cylindrical specimens $(10 \mathrm{~cm} \times 20 \mathrm{~cm})$ were produced which were subjected to natural carbonation during the periods of $14,28,56$ and 84 days, in order to evaluate the behavior of the advance of carbonation. The analyzed results demonstrate that the values obtained by the interpolation of the models proposed by different authors 
present a superior approximation in relation to the use of isolated models and present a maximum distance in relation to the real values below $20 \%$.

Keywords: durability, carbonation, prediction, efficiency.

\section{INTRODUÇÃO}

O processo de carbonatação pode ser entendido como a reação de materiais carbonatáveis do interior do concreto com os agentes agressivos atmosféricos. O produto final da reação é a formação de sais de carbonato de cálcio no interior dos poros do concreto provenientes da reação do gás carbônico com os materais alcalinos, como o hidróxido de cálcio, que carbonata em maior grau, e aluminossilicato de cálcio hidratado, que carbonata em menor grau (WOYCIECHOWSKI, 2013). A formação de sais garante rigidez superficial ao concreto, obstrução dos poros e seu processo corrosivo é caracterizado pelo desenvolvimento de trincas (ROY, POH e NORTHWOOD, 1999).

O fenômeno é regido pelo processo de difusão do agente agressivo para o inteior do concreto, proporcionando migração do local de maior concentração para o de menor concentração. Desse modo, quanto maior a concentração de $\mathrm{CO}_{2}$, mais intenso será o processo de avanço do agente agressivo, sendo que para áreas urbanas a concentração é de 0,04\% e variando até $0,3 \%$ para áreas industriais (VAN GEMERT, 2012).

No entanto, as características afetadas do concreto no processo de carbonatação são inicialmente de cunho químico, como a redução do $\mathrm{pH}$ proveninente do consumo dos materais alcalinos, fazendo com que as variações das propriedades do concreto sejam visíveis apenas quando a concentração de $\mathrm{CO}_{2}$ está alta (KOBAYASHI, SUZUKI e UNO, 1994).

Pelo fato do processo reduzir os valores de $\mathrm{pH}$ no interior dos poros concreto de 12,6 para valores inferiores a 9 , ocasionará o rompimento da pelicula passivadora do aço, proporcionada pelo ambiente ácido que causará corrosão uniforme (PAPADAKIS, FARDIS e VAYENAS, 1992). Sendo desencadeada caso exista uma quantidade significativa de água nos poros do concreto e uma diferença de potencial eletroquímico entre as regiões da armadura (HELENE, 1986), fenômeno do qual é responsável por cerca de $14 \%$ a $64 \%$ das ocorrências de degradação precoce das estruturas (ANDRADE; DAL MOLIN, 1998) apud (FÉLIX, 2017).

O avanço do agente agressivo é influenciado pelas características do meio na qual está inserido e pelas caracterísicas de dosagem, sendo os fatores do meio inserido a concentração de $\mathrm{CO}_{2}$, uma vez que em alta concentração pode reagir com outros sólidos não hidratados, intensificando o processo (PAULETTI, 2004), sendo que a concentração do gás tende a ser superior em ambientes confinados isentos de renovação de ar, plantas ou seres humanos (KAZMIERCZAK, 1995).

Em relação à umidade relativa e à temperatura, ambos os agentes trabalham conjuntamente, visto que a umidade favorece o transporte do dióxido de carbono no interior do concreto e proporciona a dissolução do gás para reagir com os demais componentes (FELIX, 2015). Além disso, a temperatura age como intensificadora da velocidade de reação que, dependendo dos valores apresentados, pode aumentar a mobilidade das células facilitando o transporte de subtâncias (TUUTTI, 1982).

Em vista de mensurar o mecanismo de avanço da carbonatação, diversos pesquisadores descrevem equações que formulam o avanço do agente agressivo. As formulações, com o passar do tempo, foram ganhando novos fatores contribuintes para a determinação do resultados finais, demonstrando grande diferença entre os dados de entrada estabelecidos pela equação de predição porposta por Tuutti (1982) e Possan (2010).

No entanto, independente dos fatores estipulados como primordiais para os resutados, boa parte das equações hoje propostas trazem o princípio descrito por Tuutti (1982), que a penetração do gás carbônico em milímetros se dá pelo produto entre a difusividade do $\mathrm{CO}_{2}$, que pode ser estipulado por investigações estruturais e pela raiz do tempo de exposição em anos. Essa simplificação é possível uma vez que se pode combinar os parâmetros do material com os do ambiente de exposição por conta do equilibrio de $\mathrm{CO}_{2}$ entre o meio externo e o meio interno, confirmando que o fenômeno obedece a primeira lei de difusão de Fick, fazendo com que todos os fatores contribuentes das demais equações sejam referentes ao valor de difusividade.

Uma grande problemática em relação as formulações é a possibilidade destas apresentarem resultados diferentes frente aos valores reais. Isso ocorre pelo fato das formulações serem de cunho determinístico e não probabilístico, onde não há intervalos a serem considerados (RIGO, OLIVEIRA e POSSAN, 2018). Outra problemática é que as formulações não consideram as modificações climáticas e modificações fisico-químicas do concreto, fazendo com que erros iniciais 
sejam projetados para predições futuras. Desse modo, a diferença em relação aos valores reais é que os sistemas equacionários não levam em consideração a determinação dos efeitos de dipersão dos parâmetros de influência referentes à durabilidade do concreto (POSSAN, 2010).

Posto as dificuldades de determinação do efeito patológico, Carmona (2005) conclui que inadequações de resultados determinísticos se dão pela falta de consistência teórica, falta de calibração das equações e pouco conhecimento em relação às mudanças de agressividade ambiental em relação às localidades que a estrutura está inseridas. SILVA et al. (2019) reforçam a afirmação ao demostrar que para simulações de idades elevadas algumas equações não atendem aos parâmetros de desempenho mínimo estipulados pela NBR 6118 (ABNT, 2014), descrevendo variações mínimas de $50 \%$. Dessa forma, o objetivo desse trabalho é propor de modo experimental uma análise da eficiência dos resultados de interpolação das modelagens matemáticas de carbonatação apresentadas por Possan e Bob e Affana em relação aos dados empíricos de profundidade de carbonatação para os intervalos de 14, 28, 56 e 84 dias, visando um método probabilístico de mensuração do fenômeno da carbonatação com poucas variações e mais próximo dos valores reais.

\section{MATERIAIS E MÉTODOS}

A dosagem adotada para a produção dos corpos de prova foi a da Associação Brasileira de Concreto Portland (ABCP) que tem como metodologia o uso das informações de massa específica, granulometria e diâmetro máximo dos agregados como base para alcançar a resistência desejada. Para este experimento se estabelece resistência à compressão de $30 \mathrm{MPa}$ aos 28 dias e abatimento do tronco de cone, conforme NBR NM 67 (ABNT, 2009), igual a $30 \pm 10 \mathrm{~mm}$. No entanto, a resistência à compressão efetiva, de acordo com ensaio preconizado pela NBR 5739 (ABNT, 2018), foi de 29,3 MPa. Os consumos dos materiais para a produção do concreto estão especificados na Tabela 1.

Tabela 1 - Consumo em $\mathrm{kg} / \mathrm{m}^{3}$ dos materiais utilizados para a fabricação das amostras

\begin{tabular}{ccccc}
$\begin{array}{c}\text { TRAÇO UNITÁRIO } \\
\text { EM MASSA }\end{array}$ & Cimento & Areia & Brita & Água \\
\cline { 1 - 3 }$\left(\mathbf{k g} / \mathbf{m}^{3}\right)$ & & & & \\
\hline $1: 1,52: 2,36: 0,47$ & 444,2 & 675,2 & 1048,3 & 208,8 \\
\hline
\end{tabular}

O cimento utilizado foi o CPII-E-32 e o agregado miúdo empregado para a produção das amostras foi uma areia quartzosa. A partir da sua distribuição granulométrica foi possível caracterizar o agregado supracitado quanto às suas propriedades físicas, seguindo especificações da NBR NM 248 (ABNT, 2003), apresentando módulo de finura (MF) de 2,38. A areia foi seca e peneirada, utilizando apenas o material passante na peneira 4,75 para a produção do concreto.

Utilizou-se como agregado graúdo uma brita comum de origem basáltica, na qual foi seca até constância de massa e após o ensaio de granulometria, conforme NBR NM 248 (ABNT, 2003), apresentou dimensão máxima característica de $6,3 \mathrm{~mm}$, conforme curva granulométrica dos agregados na Figura 1.

Figura 1 - Distribuição granulométrica dos agregados (a) miúdos e (b) graúdos.
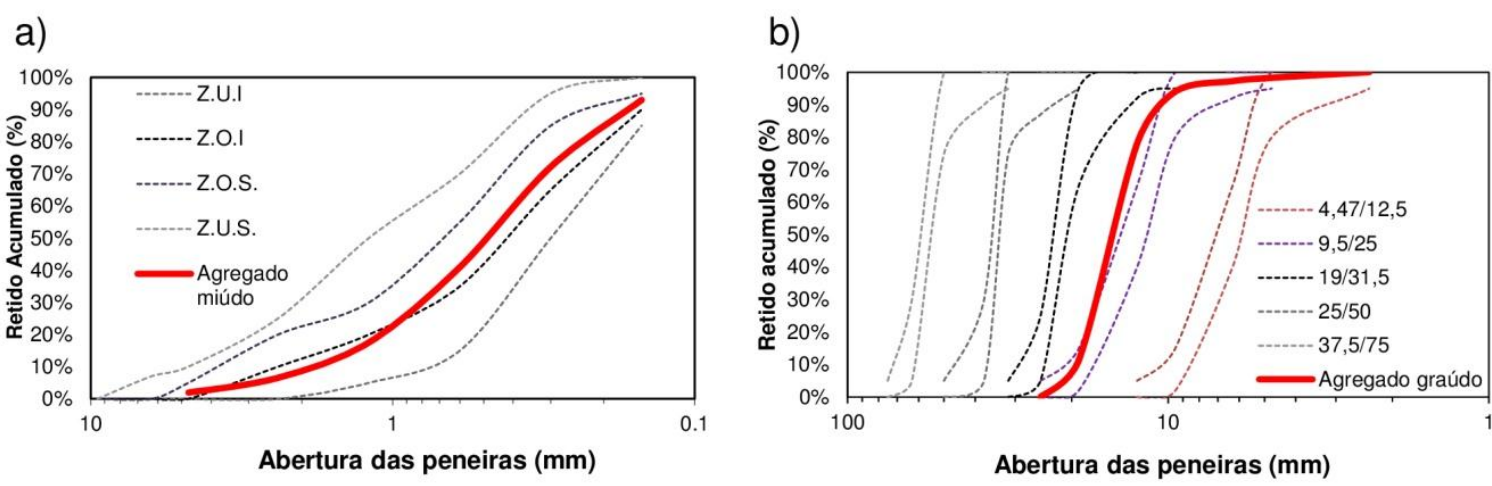

Os corpos de prova cilíndricos $(10 \mathrm{~cm}$ x $20 \mathrm{~cm})$ foram moldados e submetidos à cura submersa por 28 dias. seguindo as especificações da NBR 5738 (ABNT, 2015). Após esse período, os corpos de prova foram submetidos ao processo de carbonatação natural para os respectivos intervalos de 14, 28, 56 e 84 dias, a fim de avaliar a velocidade da penetração 
do $\mathrm{CO}_{2}$ nas idades iniciais. Uma vez que a carbonatação é um efeito com progressão potencial e não linear, a configuração estipulada inicialmente tende a manter sua angulação até atingir um valor constante de penetração ao longo do tempo, fazendo com que os dados iniciais sejam de grande importância para determinações futuras.

\subsection{Equações em análise}

As equações apresentadas foram escolhidas com o intuito de demonstrar a diferença de métodos e fatores empregados ao longo do tempo para a predição de carbonatação. Nesse trabalho, foram utilizadas as seguintes equações: Tuutti (1982) na Equação 1, que propõe que a profundidade de carbonatação é dada por um fator de difusividade K, onde o mesmo atribui valores referentes à difusividade do oxigênio; Vesikari (1988) na Equação 2, que busca determinar o valor de K a partir da relação água/cimento empregada para a produção do concreto e atribui um termo intensificador para ambientes externos sem proteção; Bob e Afanna (1993) na Equação 3, que usa como fatores determinísticos da difusividade do gás carbônico a resistencia à compressão do concreto, umidade relativa do ar, o tipo de cimento empregado no traço e a concentração de $\mathrm{CO}_{2}$ presente no ambiente e; Possan (2010) na Equação 4, que apesar de empregar todos os outros parâmetros já apresentados, como ação da umidade e quantidade de gás carbônico, esses fatores sofrem influência do tipo de cimento e das adições pozolânicas presentes no concreto, possuindo valores referentes a uma equação do tipo exponencial. As equações podem ser visualizadas abaixo.

$$
\begin{gathered}
y=k \sqrt{t} \\
k=\left[26 \cdot(a c-0,3)^{2}+1,6\right] \\
y=150 \cdot\left(\frac{c \cdot k \cdot d}{f c}\right) \cdot \sqrt{t} \\
y=k_{c} \cdot\left(\frac{20}{f c}\right)^{K f c} \cdot\left(\frac{t}{20}\right)^{\frac{1}{2}} \cdot \exp \left[\left(\frac{K a d \cdot a d \frac{3}{2}}{40+f c}\right)+\left(\frac{K C o 2 \cdot C o 2^{\frac{1}{2}}}{60+f c}\right)-\left(\frac{K R U \cdot(U R-0,58)^{2}}{100+f c}\right)\right] \cdot k_{c e}
\end{gathered}
$$

\subsection{Profundidade de carbonatação}

Em vista que o meio de exposição representa forte influência sobre o avanço da carbonatação, foram registrados os valores médios equivalente a umidade relativa do ar e a temperatura ambiente do período em que as amostras ficaram submetidas a carbonatação natural em atmosfera urbana em ambiente externo e sem proteção à interpéries, com os quais foram obtidos pelo site de climatologia (climatempo) conforme a Tabela 2.

Tabela 2 - Variação da umidade relativo do ar e da temperatura dos meses de exposição

\begin{tabular}{ccccc}
\hline \multirow{2}{*}{ Parâmetro } & \multicolumn{4}{c}{ Meses de exposição (2019) } \\
& Agosto & Setembro & Outubro & Novembro \\
\hline Umidade relativa $(\%)$ & 72 & 72 & 69 & 70 \\
Temperatura $\left({ }^{\circ} \mathrm{C}\right)$ & 26,5 & 26,5 & 27,5 & 27,5 \\
\hline
\end{tabular}

A medição dos dados reais de profundidade de carbonatação para os intervalos propostos foram realizados pelo método do paquimetro aferido, na qual após as amostras serem submetidas ao processo de carbonatação natural aspergiu-se o indicador ácido-base no interior do corpo de prova rompido diametralmente, na qual foi utilizado a solução de fenolftaleína $1 \%$, dissolvida em $70 \%$ de álcool etílico e $30 \%$ de água destilada. As amostras aspergidas descansaram por cerca de meia hora para que o indicador seja absorvido pelo corpo de prova. As medições foram feitas ao longo do comprimento da amostra, sendo efetuadas 20 medições em cada lado, totalizando 40 valores de profundidade de carbonatação estabelecidos pela diferença colorimétrica em milimetros entre a borda da amostra e o interior do concreto, de acordo com a métodos de medição descritos nas Figuras 2 e 3. 
Figura 2 - Marcação para medição da profundidade de carbonatação

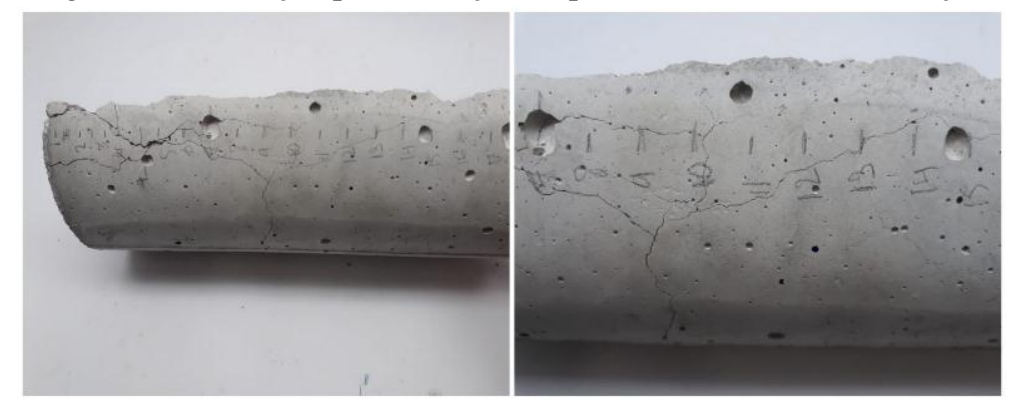

Figura 3 - Medição da profundidade de carbonatação

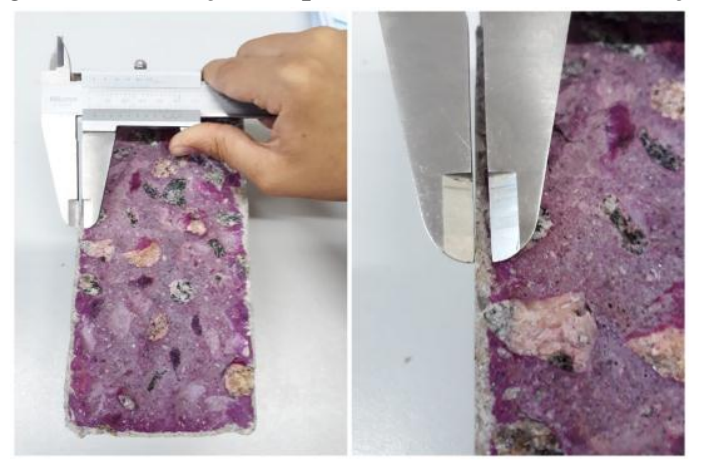

\section{RESULTADOS E DISCUSSÕES}

Ao simular os resultados de carbonatação em relação ao intervalo analisado de 84 dias, é notório que há um desvio apresentado pelas formulações que, pelo fato do avanço da carbonatação ter comportamento potencial no mecanismo de penetração, a angulação apresentada no início tende a se manter e variar proporcionalmente sem que haja qualquer desvio fora de curva, mas para condições onde não há outros fatores degradantes que influenciem na velocidade de penetração do agente agressivo. Os valores equacionários tem variação mínima de $50 \%$ em relação aos valores reais, fazendo com que no primeiro momento as formulações se apresentem inadequadas para análises de predição. A Figura 4 apresenta o gráfico de profundidade de carbonatação para o intervalo analisado.

Figura 4 - Profunfidade de carbonatação aos 84 dias

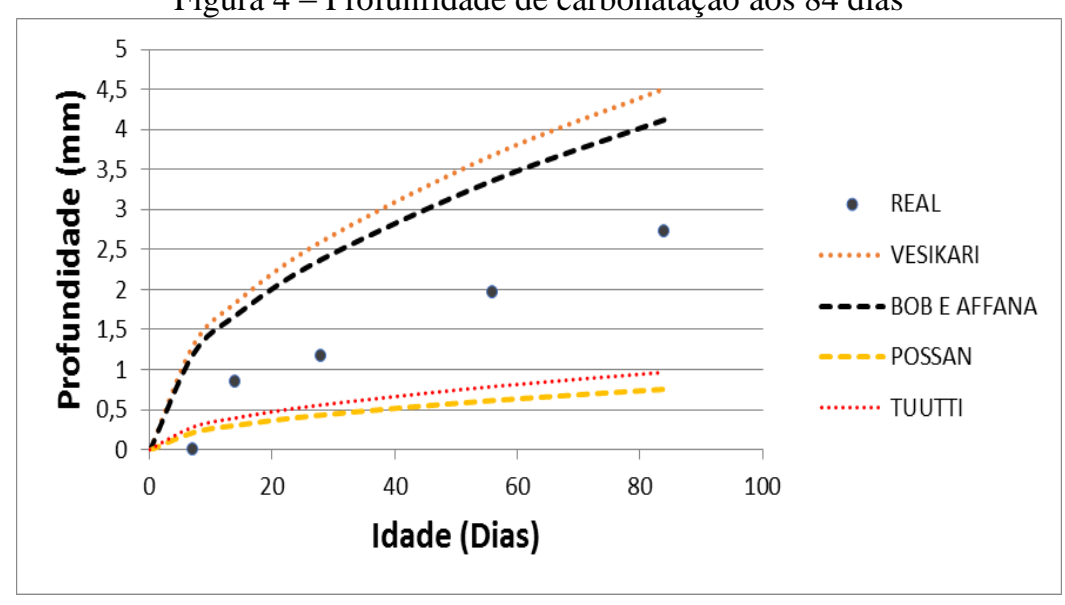

No entanto, as variações referentes aos valores apresentados também são observadas no levantamento de dados dos valores reais, demostrando, um desvio de $64 \%$ em relação ao valor médio aos 14 dias. Porém, tanto para resultados empíricos quanto para resultados equacionários, os desvios inicias são superiores aos desvios de idades mais avançadas, fazendo com que quanto maior a profundidade de carbonatação, menor o desvio apresentado. Logo, o desvio de $50 \%$ 
apresentado pelas modelagens em relação aos valores reais encontra-se dentro do intervalo de desvio padrão apresentado empiricamente, justificando a inadequação das formulações para dados iniciais. Essa variação é observada mais facilmente nas equações de Possan e Bob e Affana, pois essas duas predições dispõem de mais dados de entrada relevantes. As Tabelas 3 e 4 demostram os valores de profundidade e desvios apresentados para os valores reais e para os valores de modelagem.

Tabela 3 - Valores e desvios dos dados reais

\begin{tabular}{c|c|c|c}
\hline \multicolumn{4}{|c}{ Medição dos valores reais } \\
\hline Idade (dias) & Média $(\mathrm{mm})$ & Desvio $(\mathrm{mm})$ & Percentual de desvio \\
\hline 14 & 0,8475 & 0,54159664 & $64 \%$ \\
\hline 28 & 1,165 & 0,488613948 & $42 \%$ \\
\hline 56 & 1,9675 & 0,76736615 & $39 \%$ \\
\hline 84 & 2,7225 & 0,9096315 & $33 \%$ \\
\hline
\end{tabular}

Tabela 4 - Varição das modelagens

\begin{tabular}{c|c|c}
\hline \multicolumn{3}{|c}{ Variação das Modelagens } \\
\hline Idade (dias) & Bob e Affana & Possan \\
\hline 14 & $157 \%$ & $61 \%$ \\
\hline 28 & $114 \%$ & $46 \%$ \\
\hline 56 & $52 \%$ & $23 \%$ \\
\hline 84 & $21 \%$ & $15 \%$ \\
\hline
\end{tabular}

Como a equação proposta por Possan dispõe de mais parâmetros, os resultados finais podem ser considerados equivalentes aos dados reais quanto às variações. Além da diminuição dos valores de desvio, o avanço da carbonatação diminui a sua intensidade ao passo que aumenta a penetração do agente agressivo. Esse comportamento é observado em todas as equações propostas e caracteriza uma curva de crescimento potencial na qual tende a uma estabilidade. Esta estabilidade é observada quando a simulação da profundidade de carbonatação por equações de predição atinge 980 dias, visto que a variação é constante a partir desse dado momento, então, qualquer equação de cunho determinístico deve ser testada e validada dentro desse intervalo.

Desse modo, há uma diminuição das variações e do crescimento do efeito patológico ao longo do tempo de exposição. A partir de uma linha de tendência é possível observar o percentual de variação para intervalos posteriores em relação aos dados reais. Esse parâmetro pode ser adotado uma vez que os desvios reais são superiores aos preditos, podendo assim analisar o quanto a profundidade do agente agressivo pode variar em relação à idade da estrutura, de acordo com o proposto na Tabela 5 .

Tabela 5 - Variação da carbonatação para idades distintas

\begin{tabular}{c|c}
\hline \multicolumn{2}{c}{ Variação da Profundidade de Carbonatação } \\
\hline Idade da Estrutura & Variação \\
\hline até 6 meses & $64 \%$ a $25 \%$ \\
\hline de 6 meses a um ano & $24 \%$ a $20 \%$ \\
\hline de 1 ano a 2 anos e meio & $19 \%$ a $15 \%$ \\
\hline de 2 anos e meio a 5 anos & $14 \%$ a $12 \%$ \\
\hline de 6 anos a 8 anos e meio & $11 \%$ a $10 \%$ \\
\hline de 9 anos a 11 anos e meio & $9 \%$ \\
\hline de 12 anos a 17 anos & $8 \%$ \\
\hline de 18 anos a 25 anos e meio & $7 \%$ \\
\hline de 30 anos a 41 anos e meio & $6 \%$ \\
\hline idades superiores a 41 anos e meio & $5 \%$ \\
\hline
\end{tabular}

Desta forma, o valor atribuído para uma certa idade deve ser obervado quanto ao grau de variação, para que não sejam estipulados dados irreais. Como é conhecida a forma de variação ao longo do tempo, se fez necessário uma interpolação que possa se equiparar com os valores reais, tanto para os valores de Bob e Affana, quanto para Possan. Essa 
combinação culminou em resultados bem próximos aos reais, com desvio máximo de 20\%, como pode ser observado na Figura 5.

Figura 5 - Equação interpolada em relação aos dados reais

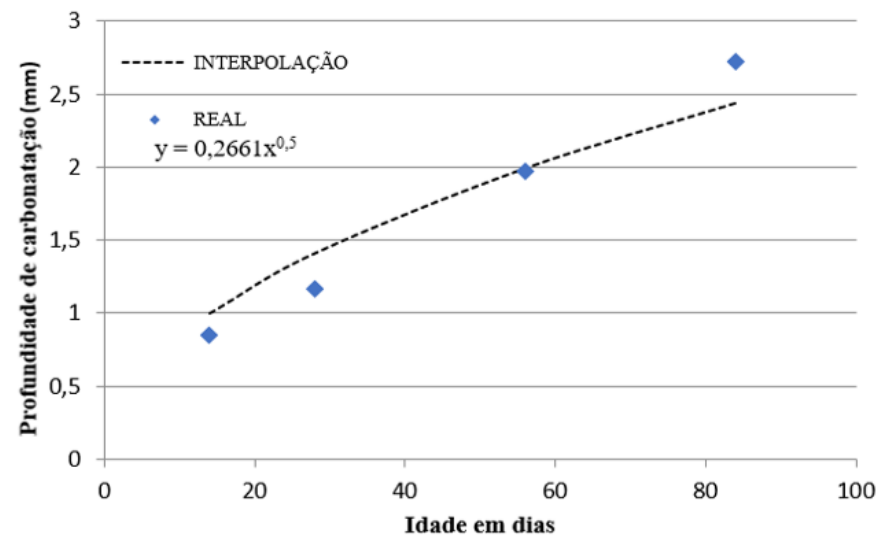

A interpolação entre as equações propostas por Bob e Affana e Possan, acrescentadas aos desvios do tempo de exposição, descrevem o crescimento da frente de carbonatação. A Figura 6 demostra o crescimento da frente de carbonatação até o invervalo de estagnação em 980 dias, descrevendo, a partir da interpolação, o mecanismo de penetração do agente agressivo.

Figura 6 - Crescimento da frente de carbonatação

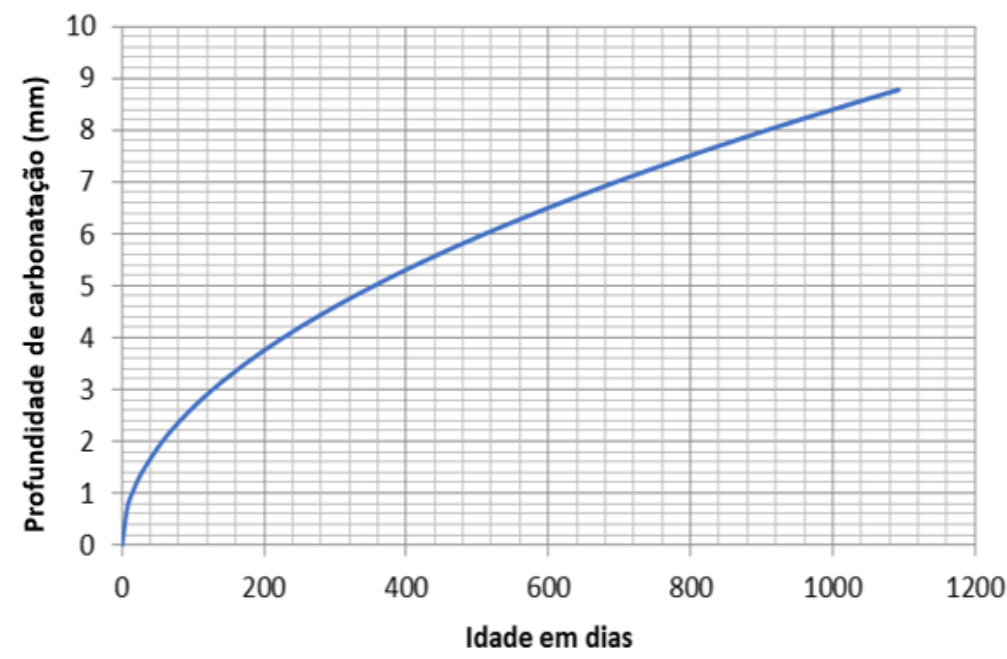

\section{CONCLUSÃO}

Mediante às analises realizadas, é possivel constatar que levantamentos de profundidade de carbonatação em idades iniciais dispõem de grande variações, podendo chegar em até 64\%, fazendo com que o intervalo analisado não seja ideal para dispor de dados para levantamentos futuros. As variações foram refletidas tanto em dados reais quanto nas equações de predição, demostrando que as formulações se apresentam como inadequadas para o intervalo analisado, ou seja, a invalidade acontece apenas onde as variações são consideráveis, logo para edificações em idades iniciais.

Uma vez alcançada certa idade, é notório que a penetração do agente agressivo tende a ser homogênea e as diferenciações apresentadas podem ser resolvidas a partir da calibração proposta por uma interpolação entre formulações ou disposição de um fator multiplicador para descrever o fenômeno.

Como as inspeções em edificações acontecem no intervalo mínimo de 5 anos, pode-se afirmar que análises realizadas em edificações com idade inferior às descritas dispõem de variações entre 64\% e 14\% que devem ser desconsideradas nas análises, fazendo com que tais dados sejam imprecisos para descrever o avanço do agente. Assim, os dados 
apresentados em idades iniciais não são satisfatórios para a determinação da profundidade de carbonatação, uma vez que as metodologias empregadas são determinístiscas e não dispõem da possibilidade probabilística apresentada pelas variações ocorrentes.

\section{REFERÊNCIAS}

ASSOCIAÇÃO BRASILEIRA DE NORMAS TÉCNICAS (ABNT). NBR NM 248: Agregados - Determinação da composição granulométrica. Rio de Janeiro, 2003.

ASSOCIAÇÃO BRASILEIRA DE NORMAS TÉCNICAS (ABNT). NBR NM 67: Concreto - Determinação da consistência pelo abatimento do tronco de cone. Rio de Janeiro, 2009.

ASSOCIAÇÃO BRASILEIRA DE NORMAS TÉCNICAS (ABNT). NBR 6118: Projeto de estruturas de concreto Procedimento. Rio de Janeiro, 2014.

ASSOCIAÇÃO BRASILEIRA DE NORMAS TÉCNICAS (ABNT). NBR 5738: Concreto - Procedimento para moldagem e cura de corpos de prova. Rio de Janeiro, 2015.

CARMONA, T. G. Modelos de previsão de despassivação das armaduras em estruturas de cocnreto sujeitas à carbontação. Dissertação (Mestrado em Engenharia Civil) - Universidade de São Paulo, São Paulo, 2005.

VAN GEMERT, D. Contribution of concrete-polymer composites to sustainable construction and conservation procedures. Restoration of Buildings and Monuments, v.18, p. 143-150, 2012.

FELIX, E. F; CARRAZEDO, R.; POSSAN, E. Análise paramétrica da carbonatação em estruturas de concreto armado via redes neurais artificiais. Revista ALCONPAT, v. 7, n. 3, 2017.

FELIX, E. F. Desenvolvimento de software para a estimativa da profundidade de carbonatação, vida útil e captura de $\mathrm{CO}_{2}$ de estruturas de concreto empregando RNA's. Trabalho de Conclusão de Curso pela Universidade Federal da Integração Latino-Americana, Paraná, 2015.

HELENE, P. R. L. Fissuração das peças de concreto armado e corrosão das armaduras. Anais do Seminário Nacional de Corrosão na Construção Civil, Rio de Janeiro, 1986.

KAZMIERCZAK, C. S. Contribuição para a análise da eficiência de películas aplicadas sobre as estruturas de concreto armado com o objetivo de proteção contra a carbonatação. Tese (Doutorado em Engenharia) Universidade de São Paulo, São Paulo, 1995.

KOBAYASHI, K.; SUZUKI, K.; UNO, Y. Carbonation of concrete structures and decomposition of C-S-H. Cement and Concrete Research, v. 24, 1994.

WOYCIECHOWSKI, P. Model of concrete carbonation. Sientific Notebooks - Buiding 157, CD-ROM, 2013.

PAPADAKIS, V. G.; FARDIS, M. N.; VAYENAS, C. G. Effect of Composition, Environmental Factors and Cementlime Motor Coating on Concrete Carbonation. Materials and Structures, v. 25, p. 293-304, 1992.

PAULETTI, C. Análise comparativa de procedimentos para ensaios acelerados de carbonatação. Dissertação (Mestrado em Engenharia) - Universidade Federal do Rio Grande do Sul, Porto Alegre, 2004.

POSSAN, E. Modelagem da carbonatação e previsão de vida útil de estruturas d econcreto em ambiente urbano. Tese (Doutorado Em Engenharia Civil) - Universidade Federal do Rio Grande do Sul, Porto Alegre, 2010.

RIGO, E., OLIVEIRA, C. E. DE, \& POSSAN, E. Aplicação de modelagem matemática para fins de previsão de vida útil de estruturas de concreto sujeitas à ação de $\mathrm{CO} 2$. Anais do $3^{\mathbf{0}}$ Simpósio Paranaense de Patologia das Construções - SPPC, p. 245-256, 2018.

ROY, S. K.; POH, K. B.; NORTHWOOD, D. O. Durability of concrete - Accelerated carbonation and weathering studies. Cement and Concrete Research, v. 34, p. 597-606, 1999.

SILVA, M.; CAVALCANTE, L.; MEDEIROS, J.; AMANCIO, F.; POSSAN, E. Eficiência dos modelos de predição da evolução da carbonatação. Anais do Concresso Brasileiro do Concreto - CBC, 61(1), Fortaleza, 2019.

TUUTTI, K. Corrosion of Steel in Concrete. Swedish Cement and Concrete Research Institute, Suecis, 1982. 